\title{
USO DE CORONAS DE ZIRCONIO EN EL TRATAMIENTO DE CARIES DE LA INFANCIA TEMPRANA
}

\section{USE OF ZIRCONIUM CROWNS IN THE TREATMENT OF CARIES OF EARLY CHILDHOOD}

\author{
Villalobos Pedro.* ${ }^{1 *}$, Mendoza Roberto. ${ }^{2}$, Yamamoto Adolfo. ${ }^{3}$ Alvear Cristina. ${ }^{4}$ \\ ${ }^{1}$ Especialista en Odontopediatría, Universidad Nacional Autónoma de México. México. \\ ${ }^{2}$ Docente Especialista en Odontopediatría, Universidad Nacional Autónoma de México. México. \\ ${ }^{3}$ Docente de la Especialidad de Odontopediatría. DEPel. Fac. de Odontología, Universidada Nacional Autónoma de México. \\ México. \\ ${ }^{4}$ Od. Esp en Odontopediatría Universidad Nacional Autónoma de México. Docente Universidad Católica de Cuenca. \\ Ecuador. \\ *pedro.va05@hotmail.com
}

\begin{abstract}
Resumen
Resumen: Las coronas de zirconio en la actualidad han creado una gran demanda, son utilizadas para el tratamiento de caries de la infancia temprana, por ello ha generado una gran controversia sobre sus efectos adversos a futuro, ya que la estética hoy en día juega un papel muy importante. El objetivo fue presentar un Caso Clínico de un paciente en dentición temporal y con diagnóstico de caries de la infancia temprana, usando como material de restauración coronas de zirconio en molares e incisivos superiores, para describir si se presentaba alguna modificación anatómica. Reporte del caso: Paciente de 3 Años 2 meses de edad que acude a la Clínica de Odontopediatría de la DEP eI de la Facultad de Odontología de la UNAM. Su motivo de consulta es referida por la madre "Caries dental y manchas en los dientes frontales". A la exploración clínica se observa dentición temporal, múltiples lesiones por caries de diversos grados. El plan de tratamiento consistió en realizar tratamientos pulpares, resinas y coronas de zirconio. Resultados: Se consiguió rehabilitar al paciente mediante restauraciones estéticas. El paciente llevará a cabo un seguimiento clínico cada 3 meses, realizando un ajuste oclusal y evaluación mediante un CONE BEAM.
\end{abstract}

Palabras clave: caries de la infancia temprana, estética, coronas de zirconio, ajuste oclusal, CONE BEAM.

\begin{abstract}
Summary: Nowadays, zirconium crowns have created a huge demand, they are used for early childhood caries treatment, that's why they have generated a lot of controversy about their future adverse effects because actually aesthetics plays a very important role. The aim was to present a clinical case of a patient in primary teeth with diagnostic of early childhood caries, using as a restorative material zirconium crowns in upper molars and incisors, to describe if any anatomical modification was presented Case report: Patient of 3 years and 2 months old who goes to the department of Pediatric Dentistry of the DEPeI of the Faculty of Dentistry, UNAM. The reason of the consultation is referred by his mother "Cavities and black spots in the front teeth." At the clinical examination it is noted primary dentition with multiple carious lesions of different degrees. The treatment plan consisted of pulp treatments, resins and zirconium crowns. Results: it was obtained to rehabilitate the patient with aesthetic restorations. The patient will have a clinical follow-up every 3 months, performing and occlusal evaluation adjustment with CONE BEAM.
\end{abstract}

Key words: early childhood caries, aesthetic, zirconium crowns, occlusal adjustment, CONE BEAM.

\section{INTRODUCCIÓN}

En la actualidad existe una gran prevalencia de la caries de la temprana infancia, la misma que ha afectado considerablemente la salud oral de la población infantil. Durante muchos años este problema se ha rehabilitado mediante tratamientos convencionales, dentro del manejo de caries dental, hoy en día la estética ha tomado importancia dentro de la odontopeditría, jugando un papel importante en el desarrollo psicológico y social del niño, sumada a esta situación la exigencia por parte de los padres. Por ello, el odontopediatra debe poseer los conocimientos sobre estos nuevos materiales o alternativas de tratamiento, considerando 
sus ventajas y desventajas; y tomando en cuenta que no todos los pacientes son candidatos para la realización de estas terapias. Sin embargo, existen diferentes variables sobre la decisión y selección de un material estético como son: las preferencias del operador, comportamiento del niño, tiempo de duración del material, el control de la hemorragia y humedad. En los últimos años las coronas de zirconio han incrementado su demanda, por lo tanto se deben considerar los efectos que pueden causarle al paciente, entre estos se han mencionado desgaste al antagonista, problemas en la articulación temporomandibular entre otros, pero en la actualidad no existen las investigaciones suficientes que demuestren lo contrario. ${ }^{1,2}$

\subsection{Caries de la Infancia Temprana.}

Se define como Caries de la Infancia Temprana (CTI), a la presencia de uno o más dientes cariados (cavitados o no cavitados), perdidos o restaurados en cualquier diente primario en un niño menor de seis años hasta los 71 meses de edad. ${ }^{1-3}$ Anteriormente era conocida como caries de biberón, pero la Asociación Americana de Odontologia Pediátrica (AAPD), adoptó el término de CTI ya que refleja su etiología multifactorial. ${ }^{3-5}$ La prevalencia de CTI en Estados Unidos es alta, en México sus cifras también son importantes, pero no se conocen sus cifras exactas; sin embargo en el año 2006-2007 se llevó a cabo una investigación en la Clínica de Odontopediatría, de la División de Estudios de Postgrado e Investigación UNAM, donde se obtuvo una prevalencia alta de $59.5 \%$ de caries de la infancia temprana en la población infantil mexicana. El tratamiento de la Caries de la Infancia Temprana en la actualidad es utilizado mediante manuales clínicos para la atención de acuerdo a la edad del paciente y los tratamientos adecuados a realizar, existen diferentes protocolos como: AAPD, sistema CAMBRA entre otros. ${ }^{6-8}$

\subsection{Zirconio}

El circonio o zirconio es un metal de transición brillante, de color blanco grisáceo, duro, resistente a la corrosión, de apariencia similar al acero. ${ }^{9,10}$ Uniendo el $\mathrm{ZrO} 2$ con otros óxidos metálicos, por ejemplo $\mathrm{MgO}, \mathrm{CaO}$, o Y2 O3, se obtiene una gran estabilidad molecular. La zirconia estabilizada con itrio, es conocida como zirconia tetragonal policristalina (TZP) y es actualmente la combinación más estudiada y utilizada en la prótesis dental con varias propiedades físicas: alta resistencia a la flexión, tenacidad a la fractura, alta dureza, excelente resistencia química y biocompatibilidad (bien adaptada a los tejidos vivos). ${ }^{11,12}$ Sus ventajas son: buena estética (translucidez), resistencia al agrietamiento, buena retención, resistencia al desgaste, excelente adhesión celular sin reacciones sistémicas, excelente durabilidad, buena satisfacción del paciente.Sus desventajas son: el costo, no se pueden ajustar fácilmente y requieren un ajuste pasivo. ${ }^{12}$

\subsection{Zirconio en Odontopediatría}

En 1947 “The Rocky Mountain Company” introdujo las coronas de acero inoxidable a la Odontología Pediátrica.

Estas restauraciones brindaron mejores resultados que otros materiales, pero por desgracia poseían pobre estética. ${ }^{13}$ En la actualidad la sociedad y los padres de pacientes pediátricos dan gran importancia a la estética lo que ha creado un nuevo desafío para los odontopediatras. Hoy en día, existen varias opciones para la restauración estética con el objetivo de llevar a cabo el tratamiento de caries de la infancia temprana, siendo las coronas de zirconio las más solicitadas por los padres de familia y la mercadotecnia generada por las empresas. ${ }^{14}$ Las propiedades de las coronas de zirconio compuestas por un dióxido de circonio cristalino, es estabilizada con óxido de itrio (3Y-TZP) obteniendo un "zirconio monolítico"; estas coronas presentan propiedades mecánicas muy similares a las de los metales, sin embargo, tienen un color similar a la de los dientes, permiten una reducción sustancial en el espesor del núcleo y son biocompatibles. ${ }^{14-16}$ Las indicaciones para el uso de las coronas de zirconio son: caries en múltiples superficies (interproximal o ángulo incisal), ${ }^{14}$ estructura dental suficiente para su retención, con una sobremordida horizontal y vertical $(1.5 \mathrm{~mm} \times 1.5 \mathrm{~mm})$, espacios fisiológicos y primate, ${ }^{16}$ pacientes cooperadores ( para el control de la humedad y hemorragia), ${ }^{17}$ defectos en la estructura dental (hipolasia o amelogenesis imperfecta), discromia dental, fractura dental, descalcificación en el tercio cervical, después del tratamiento pulpar. ${ }^{16,17}$ Las contraindicaciones para el uso de las coronas de zirconio son: pacientes con apiñamiento severo, pérdida del espacio mesio-distal severo, pacientes no cooperadores, sobremordida horizontal y vertical negativa, estructura dental insuficiente, paciente bruxista. $^{14,16,17}$

Se recomienda para la preparación de las coronas de zirconio tomar en cuenta que no se preparan igual que una corona de acero cromo y se requiere aproximadamente un $20 \%$ más de reducción, ${ }^{13,18}$ la preparación debe adaptarse a las dimensiones internas de la corona, ${ }^{18}$ realizar un buen diagnóstico pulpar antes de la colocación. Para la preparación se requiere la evaluación de la relación oclusal, observando si hay suficiente espacio, ${ }^{18,19}$ después se debe seleccionar la corona de acuerdo al ancho mesio-distal antes de la reducción del diente. Después de la cementación, hay que comprobar la oclusión; se debe realizar un ajuste oclusal en caso de ser necesario, si es necesario las coronas deben ajustarse con las fresas diseñadas específicamente de alta velocidad y con bastante irrigación, y luego pulir la corona. ${ }^{18}$ Se recomienda realizar un ajuste oclusal en la corona de zirconio de 0.25 $\mathrm{mm}$ cada tres meses y en los dientes antagonistas en caso de que así se requiera. ${ }^{18,20}$ Las coronas de zirconio hasta la actualidad existen deficiente investigaciones si causan algún efecto pero se han mencionado algunas como: daño a las superficies de oclusión antagonista, ${ }^{13,15,18}$ pérdida de la dimensión vertical, mal funcionamiento de masticación (remo- 
delación de la ATM) ${ }^{18,20}$ hipersensibilidad dental, necrosis pulpar, abrasión al esmalte, ${ }^{13}$ Sin embargo, no existen en la actualidad investigaciones confiables que comprueben dichos efectos. $^{20}$

\section{REPORTE DE CASO CLÍNICO}

Paciente masculino de 3 años 2 meses de edad. Ingresó a la clínica de Odontopediatría en la Facultad de Odontología UNAM el 14 Septiembre del 2015. Su motivo de consulta fue referido por su madre "Caries dental y manchas en los dientes frontales". A la exploración extraoral se observó un paciente con contorno de cara oval con tendencia braquifacial, implantación alta de cabello, frente amplia, buena implantación de orejas y nariz, línea media normal, ligera incompetencia labial, tercio inferior aumentado. De lado derecho se observó perfil recto, con incompetencia labial y tercio inferior aumentado. De lado derecho e izquierdo se observó perfil recto, con incompetencia labial y tercio inferior aumentado.

En la exploración intraoral se observaron mucosas hidratadas y vascularizadas, procesos óseos íntegros y continuos, dentición primaria, línea media normal, ligera mordida abierta; arcada superior oval con rugas y rafe palatino marcados, se encontraron caries dental grado 1 (esmalte) en dientes 5.5, 5.4, 5.2, 6.4, 6.5 y grado 2 (esmalte/dentina) en dientes 5.1 y 6.2 ; en la arcada inferior se observaron una implantación adecuada del frenillo lingual, arcada oval y se observaron caries dental grado 3 (esmalte/dentina/pulpa) en dientes 7.4 7.5, 8.4, 8.5. Se observó de lado derecho escalón recto con clase canina I, plano oclusal recto; de lado izquierdo escalón recto con clase canina I y plano oclusal recto. Higiene oral deficiente, con presencia de biofilm en un $21 \%$ Índice O Leary y sin desgastes fisiológicos presentes. (Figuras 1, 2, 3).

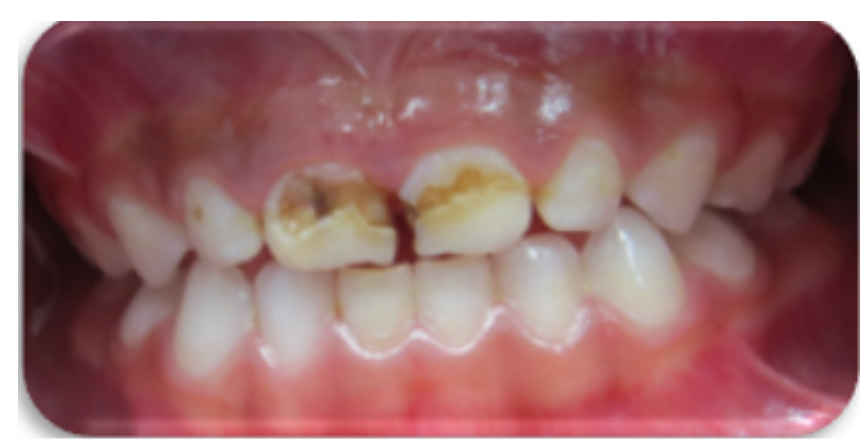

Fig. 1. Fotografía Intraoral Frontal.( Fuente propia)

Se realizó el análisis radiográfico de los dientes con mayor compromiso pulpar, los dientes anterosuperiores $5.1 \mathrm{y}$ 6.1 no se observó ningún compromiso pulpar solo caries interproximal. Se tomó radiografía de la arcada inferior derecha de los dientes 7.4 y 7.5 observando un compromiso pulpar; en la arcada inferior izquierda se tomó radiografía de los dientes 8.4 y 8.5 con compromiso pulpar. (Figuras 4A, 4B, 4C).

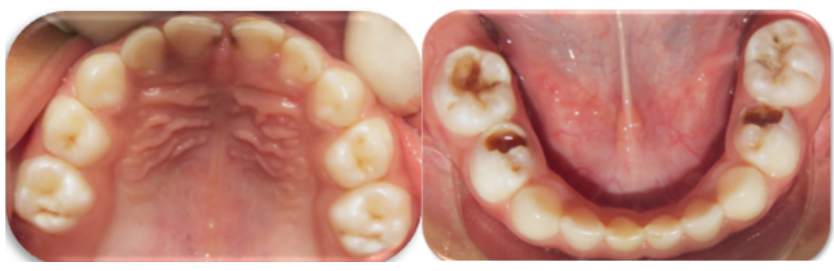

Fig. 2. Fotografía Intraoral de la Arcada Superior e Inferior. (Fuente Propia)

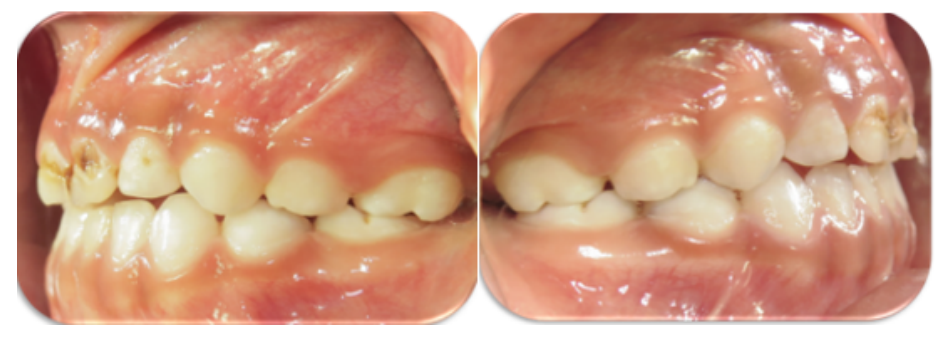

Fig. 3. Fotografía Intraoral Lateral Derecha e Izquierda. (Fuente Propia)

De acuerdo a la exploración intraoral mediante los diferentes métodos diagnósticos se obtiene un diagnóstico de Caries de la Infancia temprana por lo cual se realizó el plan de tratamiento adecuado para dicho paciente. (Tabla 1)

Se realizó la rehabilitación bucal por cuadrantes. Iniciando en el cuadrante inferior derecho donde se realizó tratamiento pulpar, al diente 8.4 pulpotomia y al diente 8.5 pulpectomia con Vitapex $\AA$; y, en el cuadrante inferior izquierdo, se realizó tratamiento pulpar a los diente 7.4 y 7.5 pulpotomias. (Figura 5)

En el cuadrante superior izquierdo se realizó la colocación de resinas en los dientes 6.4 y 6.5 ; en el cuadrante superior derecho se llevó acabo la colocación en el diente 5.4 sellador de fosetas y fisuras, en los dientes 5.5 y 5.2 resinas. Posteriormente de la cita, el paciente se remitió a la realización de un CONE BEAM, la cual es solicitada para evaluar antes de la colocación de coronas de zirconio que no exista alguna alteración. (Figura 6)

Después se llevó a cabo la colocación de las coronas de zirconio marca $\mathrm{NuSmile} \mathrm{Zr} \AA$, iniciando con la colocación del cuadrante inferior izquierdo en dientes 7.4-7.5; luego se colocaron las del cuadrante inferior derecho en dientes 8.4-8.5; y por último se realizó la colocación del cuadrante antero superior en los dientes 5.1 y 6.1. Su colocación fue

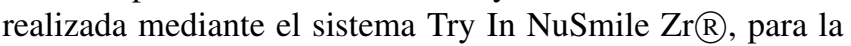
preparación de los órganos dentales en la cual se colocaron dichas coronas, fueron cementadas mediante un ionómero de

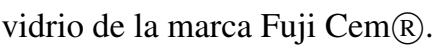

Posteriormente se realizó profilaxis con pasta, aplicación de barniz de flúor Clinpro $囚$ marca $3 \mathrm{M}$ de 22.600 ppm, técnica de cepillado, y se realizó la toma de fotografías 


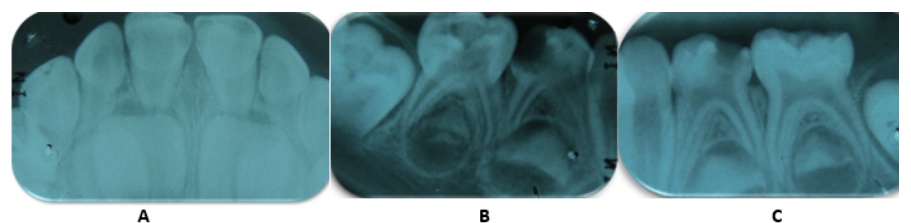

Fig. 4. A. Radiografía de la Arcada Anterosuperior. Figura 4.B Radiografía de la Arcada Inferior Derecha. Figura 4.C. Radiografía de la Arcada Inferior Izquierda. (Fuente Propia).

\begin{tabular}{|c|c|}
\hline $\begin{array}{c}\text { Arcada Superior } \\
\text { Derecha }\end{array}$ & $\begin{array}{c}\text { Arcada Superior } \\
\text { Izquierda }\end{array}$ \\
\hline $\begin{array}{l}\mathbf{5 1}=\text { Corona de } \\
\text { Zirconio + AO } \\
\mathbf{5 2}=\text { Resina } \\
\mathbf{5 3}=\text { Observación }+ \\
\text { TS } \\
\mathbf{5 4}=\text { Sellador de } \\
\text { Fosetas y Fisuras + } \\
\text { TS } \\
\mathbf{5 5}=\text { Resina + TS }\end{array}$ & $\begin{array}{l}\mathbf{6 1}=\text { Corona de } \\
\text { Zirconio }+\mathrm{AO} \\
\mathbf{6 2}=\text { Observación } \\
\mathbf{6 3}=\text { Observación }+ \\
\mathrm{TS} \\
\mathbf{6 4}=\text { Resina }+\mathrm{TS}\end{array}$ \\
\hline $\begin{array}{l}\begin{array}{c}\text { Arcada Inferior } \\
\text { Derecha }\end{array} \\
\mathbf{8 1}=\text { Observación } \\
\mathbf{8 2}=\text { Observación } \\
\mathbf{8 3}=\text { Observación + } \\
\text { TS } \\
\mathbf{8 4}=\text { Pulpotomia + } \\
\text { Corona de Zirconio } \\
+\mathrm{AO} \\
\mathbf{8 5}=\text { Pulpectomia } \\
\text { (Vitapex) + Corona } \\
\text { de Zirconio + } \\
\mathrm{AO}\end{array}$ & 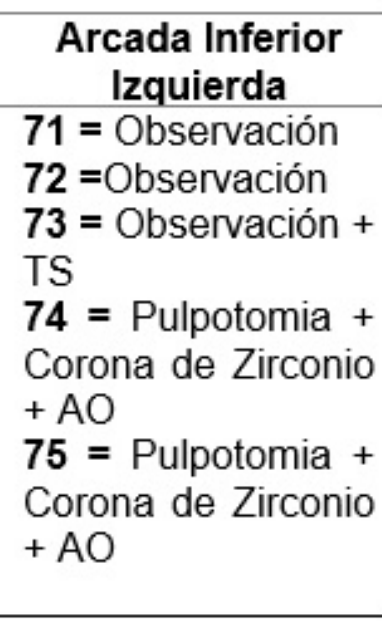 \\
\hline
\end{tabular}

Tabla 1. Plan de tratamiento de cada pieza dentaria

finales, dando las indicaciones a los familiares. (Figuras 7 , 8A)

Después de dos semanas de terminar la rehabilitación bucal, se realizó el tallado selectivo y ajuste oclusal del paciente); es remitido para tomar de nuevo el CONE BEAM para evaluar que no exista ningún efecto adverso en la cavidad oral del paciente. (Figuras 8.A; 8.B)

El seguimiento clínico del paciente de acuerdo a su nivel de riesgo a caries se llevará a cabo revisión cada 3

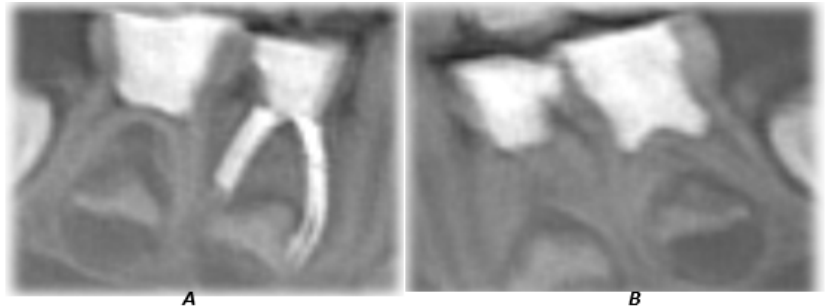

Fig. 5. A Tratamiento pulpar de los dientes 84-85. Figura 5.B. Tratamiento pulpar de los dientes 7.4-7.5. (Fuente Propia)

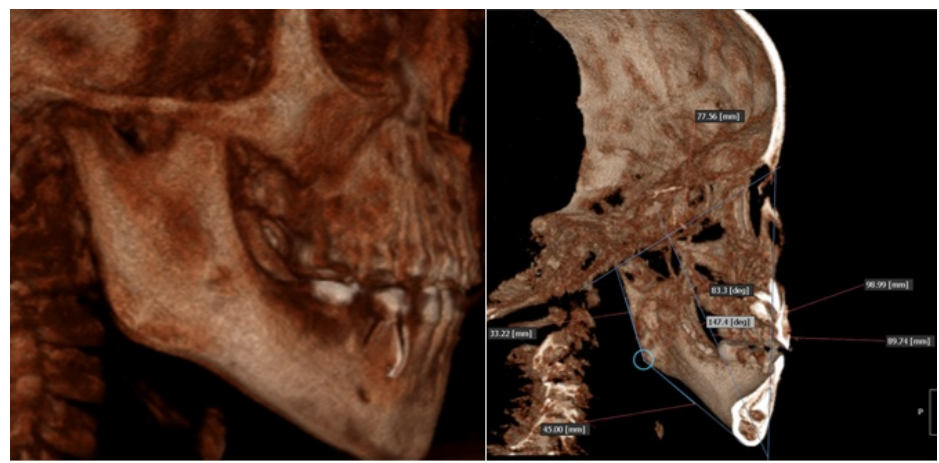

Fig. 6. A. CONE BEAM de lado derecho, donde podemos observar que no existe ninguna alteración. Se llevaron a cabo las medidas cefalométricas de los ángulos eje facial y goniaco para evaluar la posición de la mandíbula. Se obtuvo en el ángulo del eje facial $83,3^{\circ}$ y en el angulo goniaco $147,4^{\circ}$; antes de la colocación de las coronas de zirconio. Fig 6.B CONE BEAM lateral (Fuente Propia)

meses realizando control de biopelicula, profilaxis, barniz de flúor, técnica de cepillado, evaluar el tallado selectivo y ajuste oclusal; cada 6 meses tomar radiografías de control.

Tres meses después se llevó a cabo el seguimiento clínico del paciente, realizando el análisis oclusal del paciente con tallado selectivo y ajuste oclusal; observando en el diente 5.4 que la restauración colada había sido desalojada por lo cual se decide su colocación de nuevo.

Seis meses después se llevó a cabo el segundo seguimiento clínico del paciente, realizando el análisis oclusal del paciente con tallado selectivo y ajuste oclusal; se puede observar después de 6 meses que no existe ningún problema con las coronas, con buena adaptación periodontal. (Figuras 9.A, 9.B)

De acuerdo al seguimiento de evaluación mediante el CONE BEAM se remite al paciente para su realización. (Figura 10)

\section{DISCUSIÓN}

El aumento en la demanda de tratamientos estéticos en la odontopediatría ha llevado al desarrollo de coronas pediátricas a base de zirconio. De acuerdo a los fabricantes estas poseen excelentes propiedades estéticas y funcionales. ${ }^{14} \mathrm{Sin}$ 


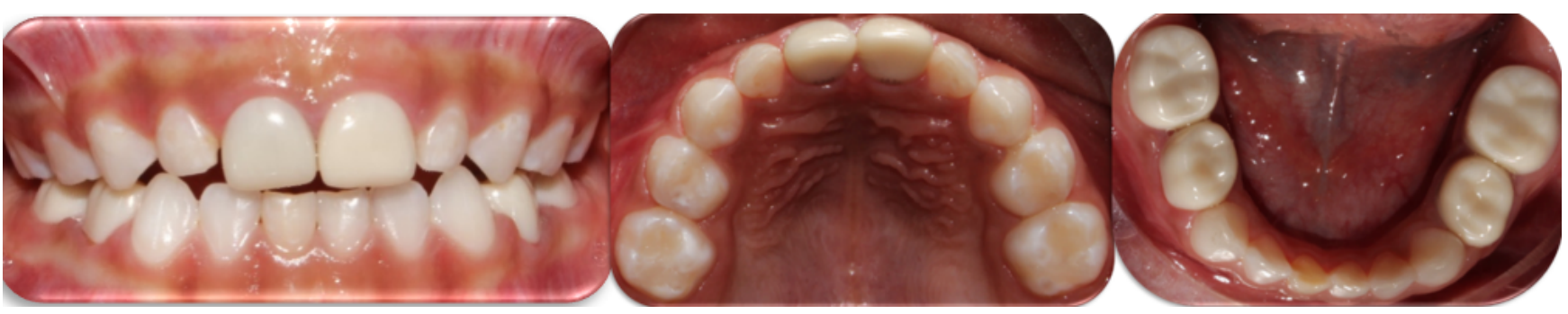

Fig. 7. Fotografías Finales; Vista Frontal y vista de las Arcadas Superior e Inferior. (Fuente Propia).
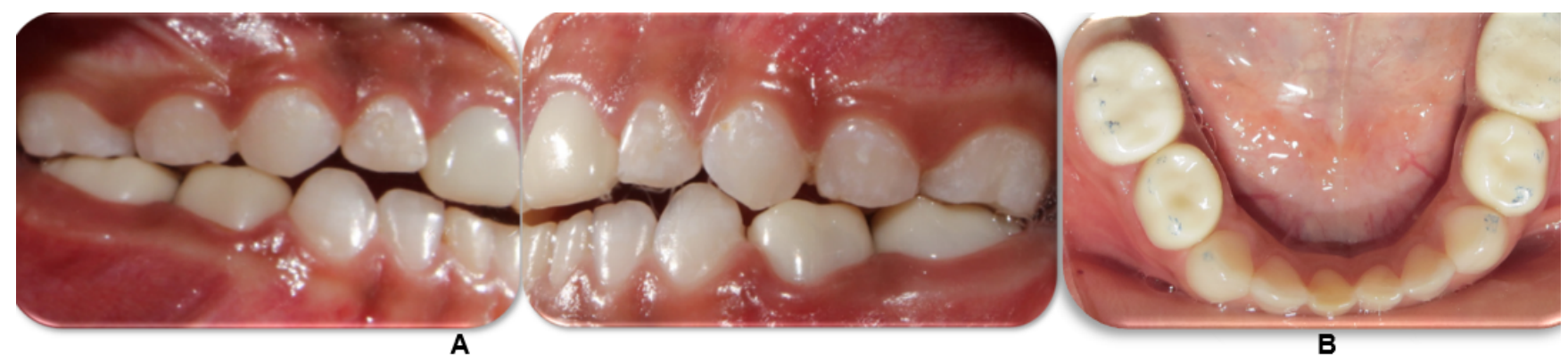

Fig. 8. A Fotografía Final Lado Lateral Derecho e Izquierdo. Fig.8.B Tallado Selectivo y Ajuste Oclusal 2 semanas posteriores.
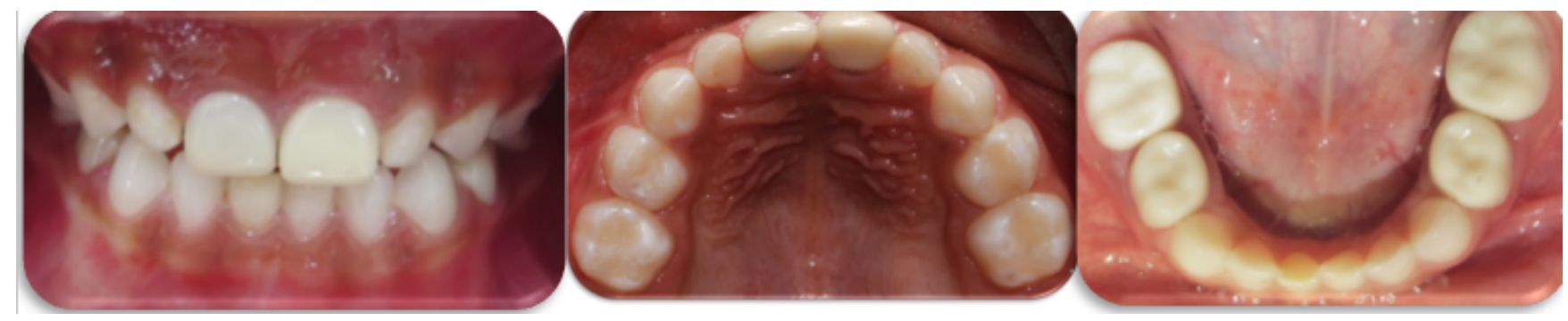

Fig. 9. A Fotografía en vista Frontal, 6 meses después. Figura 9.B Fotografía de la arcada superior e inferior 6 meses después. (Fuente Propia)

embargo, no existe evidencia contundente sobre los efectos de este producto sobre el desarrollo craneofacial, por lo que actualmente no podemos considerarla como un tratamiento de primera elección, de esta forma la selección del paciente ideal resulta fundamental. ${ }^{14,20} \mathrm{La}$ literatura menciona que el bruxismo nocturno favorece el crecimiento y desarrollo de los arcos. Sin embargo, el uso o aplicación de cualquier restauración (material) inevitablemente causará una alteración, puesto que al no poseer la misma estructura molecular sus propiedades de desgaste difieren al de la estructura dental. ${ }^{18,20}$ De acuerdo a la literatura se han realizado diferentes estudios para analizar el desgaste dental patológico. ${ }^{13-15,18}$ En un metanálisis elaborado por Mohamed cols, ${ }^{20}$ concluyó que desafortunadamente es difícil obtener buenos datos clínicos por lo cual existen pocos estudios al respecto; debido a la compleja medición directa del desgaste, obteniendo índices subjetivos y cuyos resultados llevan demasiado tiempo. ${ }^{20}$
Los resultados del paciente después de 6 meses no presentan ningún desgaste funcional del antagonista, pero se han realizado los ajustes oclusales que son recomendados cada 3 meses como es sugerido por la empresa de NuSmile $\mathbb{R} .{ }^{18}$

No obstante, se ha trabajado en estudios in vitro los cuales analizan el desgaste del esmalte después de la carga del material como es el zirconio, pero no es una reproducción exacta, ya que miden la pérdida de desgaste más no su etiología, por lo cual no son exclusivos para el desgaste mecánico. ${ }^{20}$ Sin embargo, no existen en la actualidad investigaciones que comprueben los efectos adversos generados por las coronas de zirconio. ${ }^{20}$

\section{CONCLUSIÓN}

Los resultados obtenidos en dicho paciente han sido favorables ya que el uso de coronas de zirconio cumplió el objetivo y motivo de consulta; generándole al paciente una 


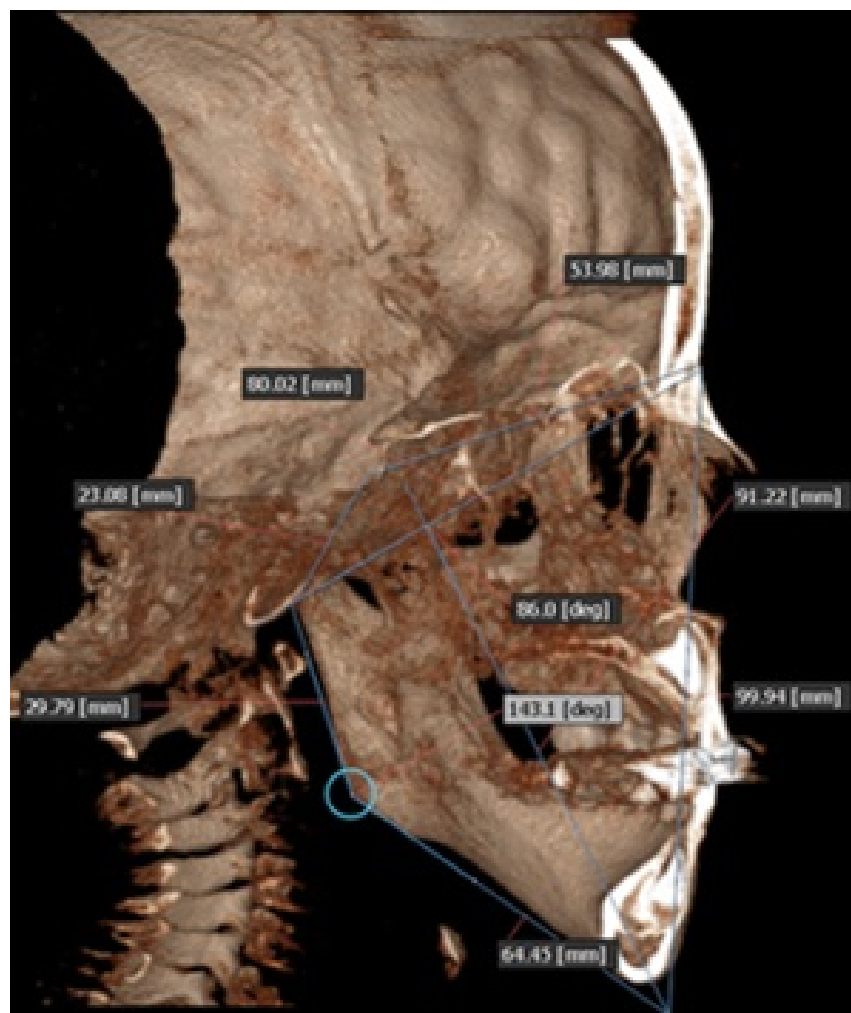

Fig. 10. CONE BEAM de lado derecho, se llevaron a cabo las medidas cefalométricas de los ángulos eje facial y goniaco para evaluar la posición de la mandíbula. Se obtuvo en el ángulo del eje facial $86^{\circ}$ y en el ángulo goniaco $143,1^{\circ}$; 6 meses después de la colocación de las coronas de zirconio, se encuentra una ligera rotación de la mandíbula que no es muy significativa. (Fuente Propia).

mejor calidad de vida gracias a la estética y funcionalidad de dicho material. De acuerdo al seguimiento clínico y evaluación mediante el CONE BEAM, no existen hasta la fecha efectos adversos. Debemos tomar en cuenta sus ventajas y desventajas, como también seleccionar de manera adecuada al paciente ya que no todos son candidatos. Se deben poseer los conocimientos sobre estos nuevos materiales o alternativas de tratamiento. El uso de coronas de zirconio en el sector anterior y posterior en pacientes pediátricos es una buena alternativa de tratamiento para la caries de la infancia temprana.

Conflicto de intereses y financiamiento Los autores declaran no tener conflicto de intereses, haber cumplido con los requisitos de autoría y haber autofinanciado este artículo.

\section{Referencias}

1 American Academy of Pediatric Dentistry. Policy on Early Childhood Caries (ECC): Classifications, Consequences, and Preventive Strategies. 2014; 37: 50-52.

2 Rojas S, Echeverria S. Caries Temprana de Infancia: ¿Enfermedad Infecciosa? 2014; 25(3):581-587.

3 Arango M, Baena G. Caries de la Infancia Temprana y Factores de Riesgo, Revisión de la Literatura. 2010; 12 (1): 59-65.

4 Kanasi E, Dewhirst F, Chalmers R. Clonal Analysis of the Microbiota of Severe Early Childhood Caries. 2010; 44: 485-497.

5 Kanasi E, Dewhirst F, Chalmers R. Clonal Analysis of the Microbiota of Severe Early Childhood Caries. 2010; 44: 485-497.

6 Barker L, Beltran E, Canto M. Surveillance for dental caries, dental sealants, tooth retention, edentulism, and enamel fluorosis. 2005; 54: 1-43.

7 Montero D, López P, Castrejón R. Prevalencia de caries de la infancia temprana y nivel socioeconómico familiar. Revista Odontológica Mexicana. 2011; 15 (2): 96-102.

8 American Academy of Pediatric Dentistry. Policy on Early Childhood Caries (ECC): Unique Challenges and Treatment Options. 2014; 37: 53-55.

9 American Academy of Pediatric Dentistry. Guideline on Caries-risk Assessment and Management for Infants, Children, and Adolescents. 2014; 37: 132-139.

10 Yasmi O, Tinanoff N, Featherstone D. Caries risk assessment, prevention, and management in pediatric dental care. Pediatric Dentistry. 2010; 12 (2): 505-517.

11 Noriega M, Karakowsky L. Caries de la infancia temprana. 2009; 23 (2): 90-97.

12 Kawashita Y, Kitamura M, Saito T. Early Childhood Caries. Int J Dent Oral Health. 2011; 23(1): 1-7.

13 Piconi C, Rimondini L, Cerroni L. El Zirconio en Odontologia. Madrid España: Editorial Amolca; 2010.

14 Pimentel J, Salazar A. Zirconia para rehabilitación completa maxilar sobre implantes. Revista Odontológica Mexicana. 2015; 19 (1): 43-47.

15 Larsson C. Zirconium dioxide based dental restorations. Studies on clinical performance and fracture behaviour. 2011; (213):9-84. 
16 Alfawaz Y. Zirconia Crown as Single Unit Tooth

Recibido: 16 de enero de 2017. Restoration: A Literature Review. J Contemp Dent Pract. 2016; 17(5):418-22.

17 Guazzato M, Proos K, Quach L. Strength, reliability and mode of fracture of bilayered porcelain/zirconia (Y-TZP) dental ceramics. 2014; 25: 5045-5052.

18 Vichi A, Louca C, Corciolani G. Color related to ceramic and zirconia restorations: a review. 2011; 27: 97-108.

19 Pokorney RL. Stainless steel preformed crowns. Rev Dent. 1965; 15: 20-6.

20 Morley J. The role of cosmetic dentistry in restoring a youthful appearance. 1999; 130: 1166-72. 
